\title{
Use of a Smartphone Application for Fracture Angulation Measurement
}

\author{
(ㄱ) İsmail Emre Ketenci, (ㄱ) Hakan Serhat Yanık
}

Department of Orthopaedics and Traumatology, Haydarpasa Numune Training and Research Hospital, Istanbul, Turkey

\begin{abstract}
Introduction: After reduction and casting of pediatric long bone fractures, angulation of the fractured segments is measured to determine if the reduction is acceptable. The aim of the present study was to assess the reliability of a smartphone application (iPinPoint) for measurement of pediatric forearm diaphyseal fracture angulations with reference to picture archive and communication systems (PACS) software measurements.

Methods: Anteroposterior and lateral forearm radiographs of 30 patients were retrospectively analyzed. Four observers measured the radius fracture angulations using the tools in the PACS software and a smartphone application. The inter- and intraobserver reliability was measured using intraclass correlation coefficients (ICCS).

Results: Very good interobserver reliability was seen among the four observers measuring the angulations on anteroposterior and lateral radiographs using the PACS software (ICC 0.962 and 0.974, respectively) and iPinPoint (ICC 0.933 and 0.959 , respectively). Intraobserver reliability was also very good for both techniques for all observers (ICC >0.9 for all observers).

Discussion and Conclusion: Both techniques are reliable in measuring fracture angulations. The iPinPoint application may represent a useful alternative to digital PACS measurements when measuring tools are not available.

Keywords: Fracture angulation; interobserver reliability; intraobserver reliability; smartphone.
\end{abstract}

$\mathrm{M}$ easurement of fracture angulation is a routine practice after closed reduction and casting of pediatric long bone fractures. Loss of correction during follow-up is also evaluated to measure the angulation, translation, and rotation of the fractured segments. Manual measurements of fracture angulations using a goniometer have largely been replaced by digital measurements that are performed using the digital tools within the picture archive and communication systems (PACS). However, patients may come with hard copy radiographs, or PACS angle measurement tools may not be available. For these cases, angle measuring smartphone applications may represent a useful alternative to digital PACS or manual goniometer measurements.

The reliability of smartphone applications has previously been assessed for Cobb angle measurements in scoliosis and kyphosis, as well as hallux valgus angle measurements [1-6]. However, to the best of our knowledge, no study has investigated the accuracy and reproducibility of a smartphone application in measuring fracture angulations. The aim of the present study was to assess the reliability of a smartphone application (iPinPoint) for measurement of pediatric forearm diaphyseal fracture angulations with reference to digital PACS measurements. Pediatric forearm

Correspondence (iletişim): İsmail Emre Ketenci, M.D. Haydarpasa Numune Egitim ve Arastirma Hastanesi, Ortopedi ve Travmatoloji Anabilim Dali, Istanbul, Turkey

Phone (Telefon): +90 2165423232 E-mail (E-posta): emreket@yahoo.com

Submitted Date (Başvuru Tarihi): 28.02.2018 Accepted Date (Kabul Tarihi): 26.07.2018

Copyright 2019 Haydarpaşa Numune Medical Journal

This is an open access article under the CC BY-NC license (http://creativecommons.org/licenses/by-nc/4.0/). 
diaphyseal fractures were selected for measurements because they are one of the most common fractures in the pediatric population that are treated with closed reduction and casting and need close monitoring during follow-up due to possible reduction loss ${ }^{[7,8]}$.

\section{Materials and Methods}

\section{Radiographic Measurements}

Conventional anteroposterior (AP) and lateral radiographs of 30 consecutive pediatric patients with diaphyseal forearm fractures who were admitted to our emergency orthopedic department between June 2017 and December 2017 were extracted from PACS records and retrospectively analyzed. Ethics Committee approval was not required since data used in our study were obtained retrospectively from patient files. All radiographs were forearm radiographs obtained by X-ray beam centered on the midshaft at a sourceto-image distance of $100 \mathrm{~cm}$, with wrist and elbow joints visible on the radiograph. For each patient, one AP and one lateral post-reduction radiographs were selected randomly from 1,2, or 3 weeks of follow-up radiographs. Only radius fracture angulations were measured to preserve the uniformity of the measurement technique and to decrease the possible measurement errors due to superimposition in lateral radiographs. Four observers, two orthopedic surgeons, and two radiologists measured the fracture angulations in AP and lateral radiographs using the PACS software of our institution and the smartphone application iPinPoint, version 2.1 (i-SmartSolutions, 2010-2017). Radiographs were presented to each observer in a random order, and the complete set of radiographs was measured by each observer using both techniques by drawing lines parallel to the long axis of fractured segments. In both techniques, the Cobb angle measurement tool was used to measure the angles between the lines. To measure the intraobserver variability, this process was conducted again after 2 weeks using the same set of radiographs in a different order to minimize the recall of prior measurements.

Smartphone measurements were performed using an Apple iPhone model 6S (Apple Inc., Cupertino, CA, USA). The iPinPoint application was downloaded free from the Apple iTunes store. It is an application that uses the built-in camera of the smartphone for angular measurement. First, a photograph was captured, pointing straight to the monitor showing the forearm radiograph. The smartphone was kept parallel to the monitor to minimize parallax errors. The Cobb angle measurement tool within the application was used to measure the magnitude of the angles similar to the

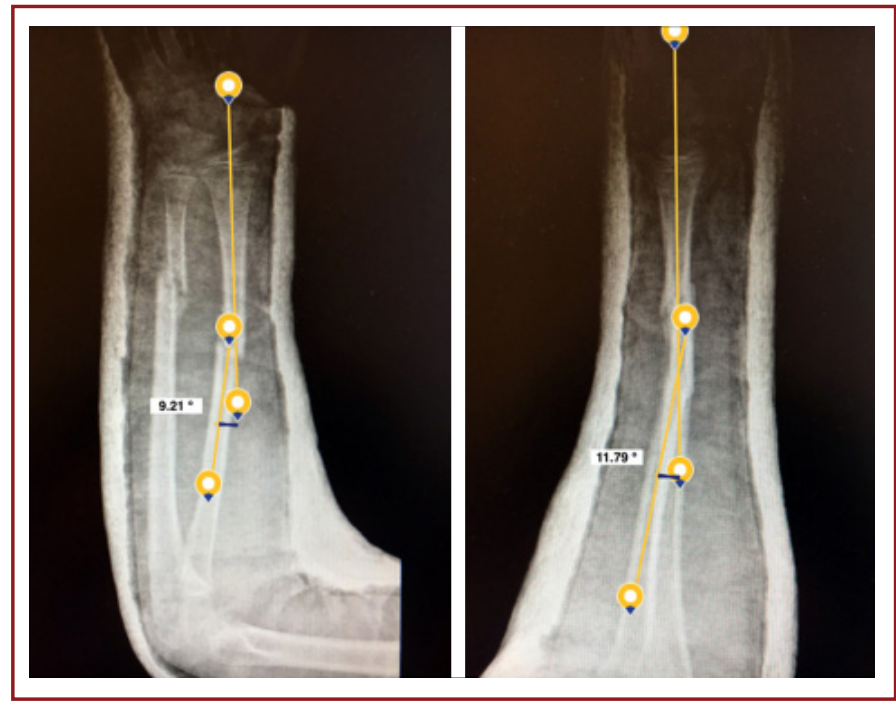

Figure 1. Measurement of radial fracture angulation in AP and lateral radiographs by iPinPoint application.

PACS measurement technique (Fig. 1). Each observer received a training period consisting of measuring 10 different forearm radiographs with the smartphone to get used to the application before the first set of measurements.

\section{Statistical Analysis}

All statistical analyses were conducted using SPSS for Windows (version 21.0; IBM Corp., Armonk, NY, USA). The interand intraobserver reliability was estimated using intraclass correlation coefficients (ICCs) for four observers. Poor reliability was considered to be present with values of $0-0.20$, fair reliability with values of $0.21-0.40$, moderate reliability with values of $0.41-0.60$, good reliability with values of $0.61-0.80$, and very good reliability with values of $0.81-1.0^{[9]}$. A p-value of $<0.05$ was considered as statistically significant.

\section{Results}

The mean age of the patients at the time of injury was 7.5 (4-10) years. Of the 30 patients, there were nine female and 21 male patients. The mean fracture angulations on AP radiographs measured by all observers were $6.1^{\circ}$ and $6.0^{\circ}$ for PACS and smartphone measurements, respectively. The mean angulations on lateral radiographs were $10.5^{\circ}$ and $10.3^{\circ}$ for PACS and smartphone measurements, respectively.

ICCs for inter- and intraobserver reliability are provided in Tables 1 and 2, respectively. Very good interobserver agreement was seen with both methods for AP and lateral plane measurements. Intraobserver reliability was also very good for both techniques for all observers. ICCs for inter- and intraobserver reliability were significant at the 0.01 level. 
Table 1. Interobserver reliability of PACS and smartphone measurements

\begin{tabular}{lcccccc}
\hline & \multicolumn{2}{c}{$\begin{array}{c}\text { PAC } \\
\text { Measurements }\end{array}$} & & & \multicolumn{2}{c}{$\begin{array}{c}\text { Smartphone } \\
\text { Measurements }\end{array}$} \\
\cline { 2 - 3 } \cline { 6 - 7 } & ICC & p & & ICC & p \\
\hline Anteroposterior & 0.962 & $<0.01$ & & 0.933 & $<0.01$ \\
Lateral & 0.974 & $<0.01$ & & 0.959 & $<0.01$ \\
\hline
\end{tabular}

Table 2. Intraobserver reliability of measurements of all observers

\begin{tabular}{|c|c|c|c|c|}
\hline & \multicolumn{2}{|c|}{$\begin{array}{c}\text { PACS } \\
\text { Measurements }\end{array}$} & \multicolumn{2}{|c|}{$\begin{array}{l}\text { Smartphone } \\
\text { Measurements }\end{array}$} \\
\hline & ICC & $\mathbf{p}$ & ICC & $\mathbf{p}$ \\
\hline \multicolumn{5}{|l|}{ Observer 1} \\
\hline Anteroposterior & 0.981 & $<0.01$ & 0.977 & $<0.01$ \\
\hline Lateral & 0.992 & $<0.01$ & 0.987 & $<0.01$ \\
\hline \multicolumn{5}{|l|}{ Observer 2} \\
\hline Anteroposterior & 0.972 & $<0.01$ & 0.963 & $<0.01$ \\
\hline Lateral & 0.985 & $<0.01$ & 0.968 & $<0.01$ \\
\hline \multicolumn{5}{|l|}{ Observer 3} \\
\hline Anteroposterior & 0.975 & $<0.01$ & 0.971 & $<0.01$ \\
\hline Lateral & 0.984 & $<0.01$ & 0.979 & $<0.01$ \\
\hline \multicolumn{5}{|l|}{ Observer 4} \\
\hline Anteroposterior & 0.942 & $<0.01$ & 0.953 & $<0.01$ \\
\hline Lateral & 0.967 & $<0.01$ & 0.979 & $<0.01$ \\
\hline
\end{tabular}

\section{Discussion}

Diaphyseal fractures of the radius and ulna are the third most common fractures in the pediatric population[7,8]. These fractures are most commonly treated non-operatively. However, close monitoring during follow-up is mandatory, since reduction loss may occur, especially in unstable fracture types. This is also true for other pediatric long bone fractures that are treated non-operatively ${ }^{[10]}$. After reduction and casting of a long bone fracture, angulation of the fractured segments is measured to determine if the reduction is acceptable. This procedure is repeated at each visit during follow-up. Different measuring options are available according to the format of the image.

Measurements on non-digital radiographs are usually performed using a goniometer. However, with the replacement of hard copy radiographs by digital images and the use of PACS software, digital measurements are more widely used nowadays. Good correlation to manual measurements and improved measurement precision has been shown for digital measurements of the hallux valgus and Cobb angles. Moreover, improved inter- and intraobserver agreements compared with manual measurements were found in some previous studies ${ }^{[11,12]}$. The integration of mobile technology into daily life introduced another option to clinical practice for radiographic angle measurements, that is, smartphone applications. They can be used to measure angles on both hard copy and digital images.

Several previous studies have been performed to assess the reliability of smartphone applications in measuring hallux valgus and Cobb angles by comparing them with manual or digital measurements ${ }^{[1-6]}$. Shaw et al. ${ }^{[1]}$ and Qiao et al. ${ }^{[2]}$ compared goniometer and smartphone measurements of Cobb angles in scoliosis and found smartphone measurements to be equivalent to manual measurements with regard to reliability and efficiency. In their study, the time consumed for smartphone measurements was significantly shorter. Ege et al. ${ }^{[4]}$ and Walter et al. ${ }^{\text {[5] }}$ compared smartphone and PACS measurements of hallux valgus angles and found that smartphone applications are as reliable as PACS software. The applications used in these studies were accelerometer based, which utilizes the position sensor of the device. The iPinPoint application, which was used in our study, makes the measurements on photographs. This application was used for the first time in the study by Dinato et al. ${ }^{[6]}$. They found iPinPoint to be more reliable than an accelerometer based application in measuring hallux valgus angles with reference to goniometer measurements.

In the present study, we tried to assess the inter- and intraobserver reliability of a smartphone application (iPinPoint) in measuring fracture angulations, which was not performed before, to our knowledge. Our hypothesis was that smartphone measurement of fracture angulations has a comparable reliability to PACS measurements, which has been shown to be a reliable technique. In our study, ICCs of both PACS and iPinPoint measurements far exceeded the threshold of 0.6 that was recommended by Chin et al. ${ }^{[13]}$ for reliable measurement. This result confirmed our hypothesis. We tested the application on radius fracture angulations, but we think that it can also be used for other long bone fractures, where the same measuring principles are used.

The present study has several limitations. Measurements on photographs captured with smartphones are prone to parallax errors. Special care should be given to position the plane of the smartphone screen parallel to the computer screen. However, there were no statistically significant differences due to this effect. Another limitation is that our reference measurement technique is also prone to variations, but PACS measurements are generally accepted as 
reliable. We also did not compare the time consumed for both measurement techniques. However, subjectively, both techniques were very fast, especially after having the training period and getting used to the application for the smartphone technique.

Measuring radiographic angles with smartphone applications has several advantages. It is a practical method that eliminates the need to perform additional equipment (a goniometer) when hard copy radiographs are available. It can also be used to measure digital radiographs when angle measuring tools are not present within the software. In conclusion, the smartphone application iPinPoint can be used as a reliable tool for measuring fracture angulations. As many surgeons have a smartphone, the use of this practical application may solve problems when angle measuring PACS tools or manual measurement equipment is not available.

Peer-review: Externally peer-reviewed.

Conflict of Interest: None declared.

Financial Disclosure: The authors declared that this study received no financial support.

Authorship Contributions: Concept: I.E.K., H.S.Y.; Design: İ.E.K., H.S.Y.; Data Collection or Processing: I.E.K., H.S.Y.; Analysis or Interpretation: I.E.K., H.S.Y.; Literature Search: I.E.K., H.S.Y.; Writing: I.E.K., H.S.Y.

\section{References}

1. Shaw M, Adam CJ, Izatt MT, Licina P, Askin GN. Use of the iPhone for Cobb angle measurement in scoliosis. Eur Spine J 2012;21:1062-8. [CrossRef]

2. Qiao J, Liu Z, Xu L, Wu T, Zheng X, Zhu Z, et al. Reliability anal- ysis of a smartphone-aided measurement method for the Cobb angle of scoliosis. J Spinal Disord Tech 2012;25:E88-92.

3. Jacquot F, Charpentier A, Khelifi S, Gastambide D, Rigal R, Sautet A. Measuring the Cobb angle with the iPhone in kyphoses: a reliability study. Int Orthop 2012;36:1655-60.

4. Ege T, Kose O, Koca K, Demiralp B, Basbozkurt M. Use of the iPhone for radiographic evaluation of hallux valgus. Skeletal Radiol 2013;42:269-73. [CrossRef]

5. Walter R, Kosy JD, Cove R. Inter- and intra-observer reliability of a smartphone application for measuring hallux valgus angles. Foot Ankle Surg 2013;19:18-21. [CrossRef]

6. Mattos E Dinato MC, Freitas MF, Milano C, Valloto E Jr, Ninomiya AF, Pagnano RG. Reliability of Two Smartphone Applications for Radiographic Measurements of Hallux Valgus Angles. J Foot Ankle Surg 2017;56:230-3. [CrossRef]

7. Truntzer J, Vopat ML, Kane PM, Christino MA, Katarincic J, Vopat BG. Forearm diaphyseal fractures in the adolescent population: treatment and management. Eur J Orthop Surg Traumatol 2015;25:201-9. [CrossRef]

8. Pace JL. Pediatric and Adolescent Forearm Fractures: Current Controversies and Treatment Recommendations. J Am Acad Orthop Surg 2016;24:780-8. [CrossRef]

9. Shrout PE, Fleiss JL. Intraclass correlations: uses in assessing rater reliability. Psychol Bull 1979;86:420-8. [CrossRef]

10. Lieber J, Schmittenbecher P. Developments in the treatment of pediatric long bone shaft fractures. Eur J Pediatr Surg 2013;23:427-33. [CrossRef]

11. Srivastava S, Chockalingam N, El Fakhri T. Radiographic angles in hallux valgus: comparison between manual and computerassisted measurements. J Foot Ankle Surg 2010;49:523-8.

12. Kuklo TR, Potter BK, Schroeder TM, O'Brien MF. Comparison of manual and digital measurements in adolescent idiopathic scoliosis. Spine 2006;31:1240-6. [CrossRef]

13. Chinn S. Statistics in respiratory medicine. 2. Repeatability and method comparison. Thorax 1991;46:454-6. [CrossRef] 